International Journal of Modern Physics A

(C) World Scientific Publishing Company

\title{
Conceptual Design Studies for a CEPC Detector
}

\author{
S.V. Chekanov and M. Demarteau \\ HEP Division, Argonne National Laboratory, 9700 S. Cass Avenue, Argonne, IL 60439, USA. \\ Emails: chekanov@anl.gov,demarteau@anl.gov
}

\begin{abstract}
The physics potential of the Circular Electron Positron Collider (CEPC) can be significantly strengthened by two detectors with complementary designs. A promising detector approach based on the Silicon Detector (SiD) designed for the International Linear Collider (ILC) is presented. Several simplifications of this detector for the lower energies expected at the CEPC are proposed. A number of cost optimizations of this detector are illustrated using full detector simulations. We show that the proposed changes will enable to reach the physics goals at the CEPC.

Keywords: e+e-, jets, Monte Carlo, CEPC

PACS numbers: 13.66.-a, 13.66.Jn
\end{abstract}

\section{Introduction}

The Circular Electron Positron Collider (CEPC) project is currently planned in China as a Higgs factory. Operating at the center-of-mass (CM) energy of $250 \mathrm{GeV}$ (or above), the CEPC experiment will take advantage of the clean environment of $e^{+} e^{-}$collisions needed for high-precision measurements of the Higgs boson. CEPC experiments can significantly strengthen our understanding of the fundamental processes at the electroweak sector of the Standard Model (SM), and can lead to discoveries of new physics through the precision measurements of the SM.

In order to achieve the physics goals at the CEPC, a detector should be well optimized for measurements of $e^{+} e^{-}$annihilation. In particular, the studies of physics processes in the Higgs sector are considered to be the primary goal of the new experiment. A promising approach for a detector at the CEPC can be based on the Silicon Detector (SiD) concept ${ }^{1}$ developed for the International Linear Collider (ILC). ${ }^{2,3}$ The design of this detector has a long history, and the experience gained during the $R \& D$ phase of this detector can be extremely valuable during the preparation to the CEPC concept.

The abbreviation "SiD" stands for "silicon detector" - a compact generalmultipurpose detector designed for high-precision measurements of $e^{+} e^{-}$annihilation at a CM energy of $500 \mathrm{GeV}$, which can be extended to $1 \mathrm{TeV}$. The choice of silicon for the tracking system and for the electromagnetic calorimeter ensures that the detector is robust to beam backgrounds, while a high-granular calorimeter 
is well suited for the reconstruction of separate particles. Some key characteristics of the SiD detector are:

(1) $4 \pi$ solid angle coverage for reconstructed particles;

(2) Full 5-layer silicon tracking system with $50 \mu \mathrm{m}$ readout pitch size;

(3) Silicon pixel detector with $20 \mu m$ readout pitch size;

(4) Superconducting solenoid with a 5 Tesla (T) field;

(5) Highly segmented silicon-tungsten electromagnetic calorimeter (ECAL) with the transverse cell size of $0.35 \mathrm{~cm}$;

(6) Highly segmented hadronic calorimeter (HCAL) with a transverse cell size of $1 \times 1 \mathrm{~cm}$. The depth of the HCAL in the barrel region is about 4.5 interaction length $^{\mathrm{a}}\left(\lambda_{I}\right)$. The calorimeter has 40 longitudinal layers in the barrel and 45 layers in the endcap region;

Both ECAL and HCAL calorimeters are finely segmented longitudinally and transversely. This is required for "imaging" capabilities of the calorimeter system: Together with the efficient tracking, the fine segmentation of the calorimeter system optimizes the SiD detector for particle-flow algorithms (PFA) which allow identification and reconstruction of separate particles. The PFA objects can be reconstructed using the Pandora Particle Flow algorithm. ${ }^{4,5}$

The response of the $\mathrm{SiD}$ detector to physics processes is simulated using the SLIC software package ("Simulator for the Linear Collider") ${ }^{6}$ developed for the ILC project. The main strength of this software lies in the fact that it can easily be configured using XML option files, and it has a platform-independent reconstruction which can be easily deployed on computers with different operating systems.

The M\&S cost of the baseline design of the $\mathrm{SiD}$ detector is estimated to be around $\$ 320 \mathrm{M},{ }^{1}$ with $32 \%$ being allocated for the calorimeter, and $37 \%$ for the magnet (estimated in 2009).

\section{SiD for the CEPC}

For the CEPC physics goals, the SiD detector is over-designed. For example, the cost can be substantially reduced by simplifying the calorimeters and by reducing the magnetic field of the solenoid. Due to the lower CM energy of $250 \mathrm{GeV}$ at the CEPC, a number of optimizations of the $\mathrm{SiD}$ detector are proposed:

(1) $5 \mathrm{~T}$ solenoid field can be reduced to $4 \mathrm{~T}$;

(2) 40 layers of HCAL can be reduced to 35 by removing 5 outer HCAL layers in the SiD design. The remaining 35 layers of the steal absorber correspond to about 4.1 nuclear interaction length;

(3) 45 layers of the HCAL endcap can be reduced to 35 layers. This makes the CEPC detector fully uniform from the point of view of the HCAL depth.

${ }^{a}$ Nuclear interaction length, $\left(\lambda_{I}\right)$, is the average distance traveled by a hadronic particle before undergoing an inelastic nuclear interaction. 
The reason for the reduction of the solenoid field lies in the fact that the typical track momentum measured at CEPC is a factor of two (four) smaller compared to the 500 (1000) $\mathrm{GeV} e^{+} e^{-}$collisions at the ILC. The magnetic field could be further reduced, but this will require a more detailed study than shown in this paper. Similarly, the reduction of the calorimeter depth is motivated by the fact that the maximum jet transverse momentum at the CEPC is $125 \mathrm{GeV}$, which is a factor two (four) smaller than for the $500(1000) \mathrm{GeV} e^{+} e^{-}$machine. In terms of the HCAL interaction length, the proposed $4 \lambda_{I}$ calorimeter is similar to that of the OPAL experiment. ${ }^{7}$ The total absorber (steal and tungsten) of the ECAL and HCAL calorimeter systems corresponds to about $5.1 \lambda_{I}$.

In order to explore the possibility of optimization of the $\mathrm{SiD}$ detector to a lower $\mathrm{CM}$ energy, we use the HepSim Monte Carlo repository ${ }^{8}$ with several benchmark processes for $e^{+} e^{-}$collisions. The $e^{+} e^{-}$events at the $250 \mathrm{GeV} \mathrm{CM}$ energy were generated using the PYTHIA $6^{9}$ model. The following processes were generated:

- Fully inclusive QCD dijet process;

- $Z$-boson production with the decays $Z \rightarrow e^{+} e^{-}, Z \rightarrow \mu^{+} \mu^{-}, Z \rightarrow \tau^{+} \tau^{-}$, $Z \rightarrow b \bar{b}$

- Higgs production $\left(Z^{0} H\right)$ with the decays $H \rightarrow 4 l, H \rightarrow \gamma \gamma, H \rightarrow \tau^{+} \tau^{-}$, $H \rightarrow b \bar{b}$. The Higgs mass was set to $125 \mathrm{GeV}$.

The events were simulated using the SiD detector geometry, and reconstructed using the SLIC package with Pandora PFA. The simulation and reconstruction steps were performed using the Open-Science Grid. ${ }^{10}$ Events before and after the simulation of the detector response were registered in the HepSim data catalogue.

In the following, the original SiD detector geometry will be called SiDloi3. The number of reconstructed events after the SiDloi3 detector simulation and reconstruction was about ten thousand. Most representative observables which are expected to be sensitive to the tracking and calorimeter performance of the SiD detector were analysed. The obtained results (not shown) were found to be within the specification of the SiD detector described in Ref. ${ }^{3}$

The same data samples were simulated and reconstructed using the CEPCoptimized geometry discussed in the beginning of this section, i.e. with the solenoid field changed from $5 \mathrm{~T}$ to $4 \mathrm{~T}$, and the HCAL calorimeter depth reduced from 40 (45) to 35 layers. In the following, the SiD geometry after such modifications called SiDcc1. Full details of this detector geometry are available from the HepSim repository. To reduce computation time, the number of simulated and reconstructed events for the SiDcc1 detector were a factor two smaller than for the SiDloi3 simulation.

The distributions of several observables which are particularly sensitive to the change in the strength of the solenoid field and the HCAL absorber depth is shown in Figs. 1 and 2. The distributions were reconstructed from the PFA objects which combine the information from four-momenta of tracks and calorimeter energy deposits. For example, the $Z$ boson mass reconstructed from the invariant mass of two electrons (Fig. 1(a)) is sensitive to the performance of tracking system to high- 


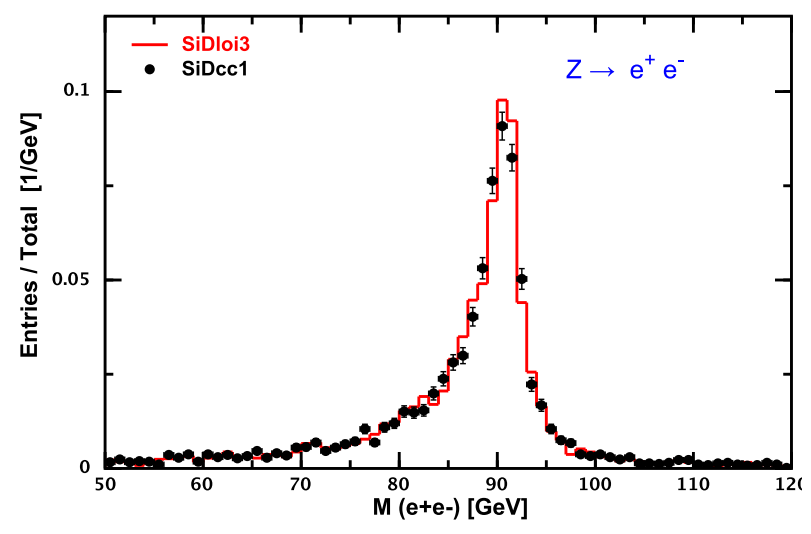

(a)

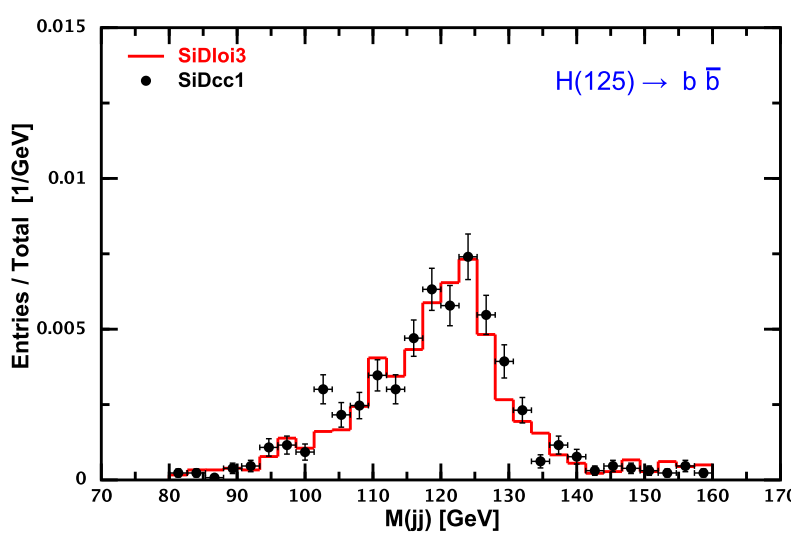

(b)

Fig. 1. The invariant mass of two reconstructed electrons for the $Z \rightarrow e^{+} e^{-}$process (a) and the invariant mass of two jets for the process $H(125) \rightarrow b \bar{b}(\mathrm{~b})$. The distributions were reconstructed from the PFA objects. The figure shows the original SiD setup (SiDloi3) and a CEPC optimized version of the $\mathrm{SiD}$ detector $(\mathrm{SiDcc} 1)$. The distributions of the latter setup are shown as solid dots with statistical uncertainties.

momentum tracks $\left(e^{+} / e^{-}\right)$. The energy distribution of hadronic jets reconstructed from the PFA objects is sensitive to both the performance of the tracking system, and to the HCAL longitudinal segmentation. Figure 1(b) shows the invariant mass of two jets for the process $H(125) \rightarrow b \bar{b}$. The jets were reconstructed with the Jade algorithm ${ }^{11}$ by forcing two jets per event, and requiring the transverse momentum of jets to be above $20 \mathrm{GeV}$.

To take a closer look at the hadronic jets, Figure 2 shows the distribution of the jet transverse momentum for inclusive QCD processes in $e^{+} e^{-}$at $250 \mathrm{GeV}$. The jets 


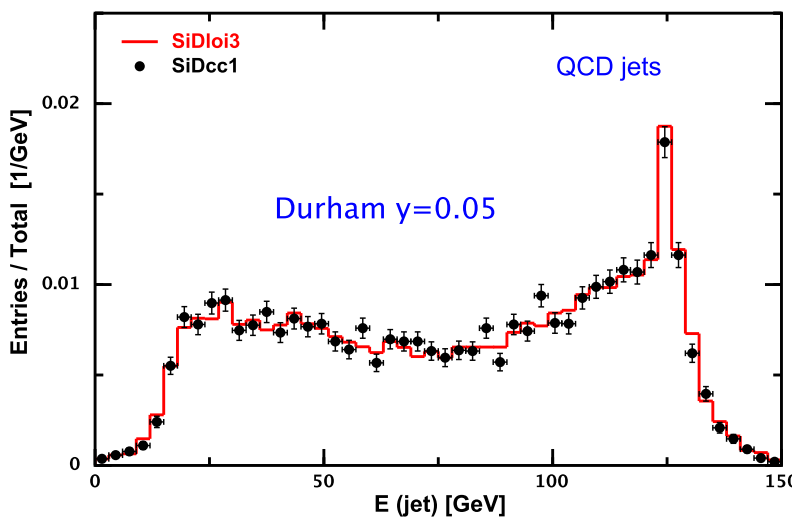

(a)

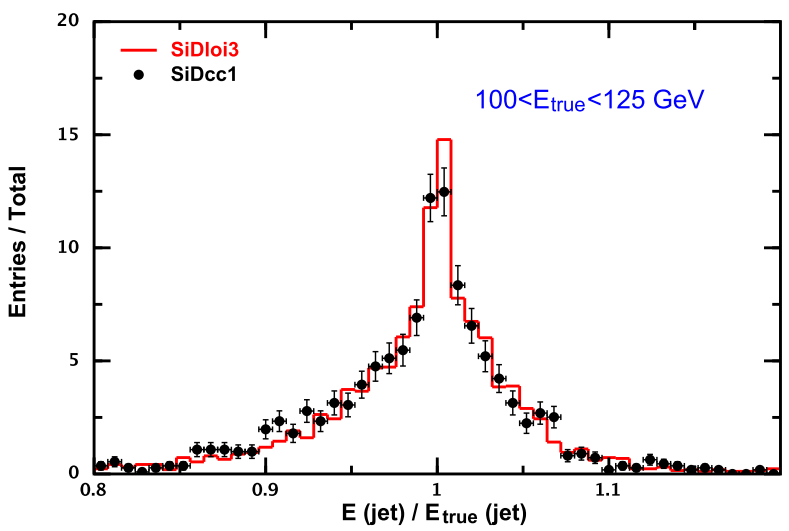

(b)

Fig. 2. The distribution of QCD jets in $e^{+} e^{-}$collisions using the Durham algorithm with $y_{\text {cut }}=$ 0.05 (a), and the jet energy response for jets with energy close to the kinematic peak of $125 \mathrm{GeV}$. The jets were reconstructed from the PFA objects. The figure shows the original SiD setup (SiDloi3) and a CEPC optimized version of the SiD detector (SiDcc1). The distributions of the latter detector setup are shown as solid dots with statistical uncertainties.

were reconstructed using the Durham algorithm ${ }^{12}$ with the parameter $y_{\text {cut }}=0.05$. As before, the input to this algorithm are the PFA objects. Jets were selected with a minimum transverse momentum of $20 \mathrm{GeV}$. Figure 2(b) shows the jet energy response by taking the ratio of the reconstructed jet energy to the energy of jets reconstructed from stable particles, which are defined if their lifetime $\tau$ are smaller than $3 \cdot 10^{-10}$ seconds. Neutrinos were excluded from consideration. As expected, the distributions for this ratio peaks at one, indicating that no energy leakage is observed for both the SiDloi3 and SiDcc1 detectors. 


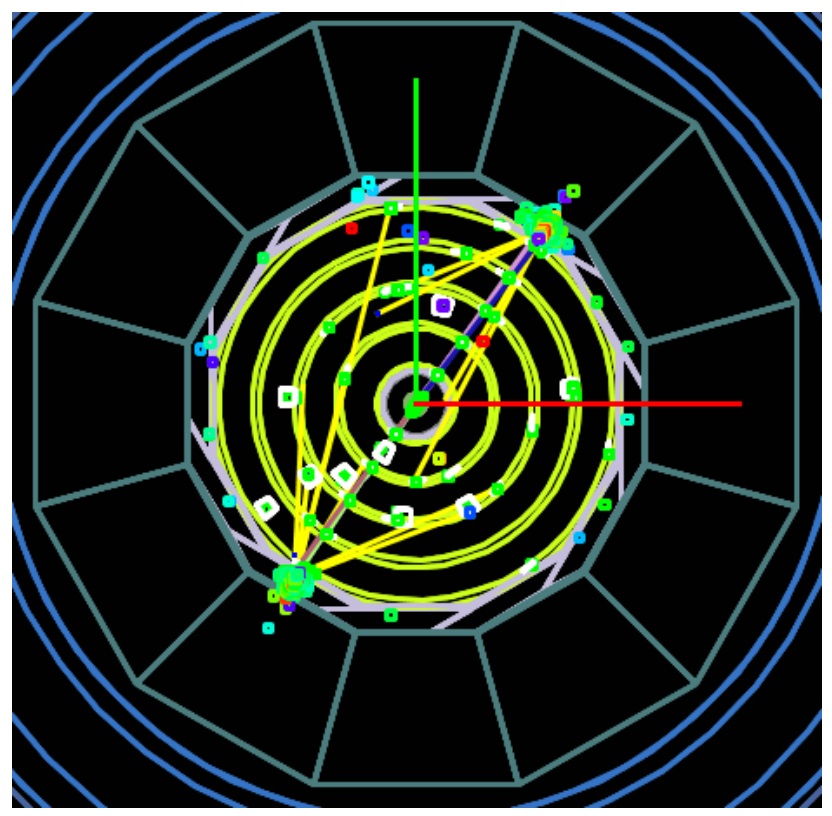

Fig. 3. An event display of an $Z \rightarrow e^{+} e^{-}$event using the optimized SiDcc1 setup. Clusters of the green points on the surface of the ECAL correspond to the reconstructed $e^{+} / e^{-}$from the $Z$ decay. The space between the outer layer of HCAL and the solenoid is due to removed 5 outer HCAL layers of the original $\mathrm{SiD}$ detector.

Figure 3 illustrates a typical $Z \rightarrow e^{+} e^{-}$event in the Jas3/Wired4 event display. A prominent feature of this event is the energy deposits in the ECAL corresponding to the electrons from the $Z$ decay. The space between the outer layer of the HCAL and the solenoid is due to the removal of 5 HCAL layers from the original design of the $\mathrm{SiD}$ detector.

In summary, this paper suggests that the $\mathrm{SiD}$ detector (or its sub-detectors) can be re-purposed for the CEPC. We have illustrated a few directions to optimize the $\mathrm{SiD}$ detector for lower $\mathrm{CM}$ energies. The results obtained with the SiDloi3 and SiDcc1 detector concepts show good agreements (within statistical errors), thus the optimized SiDcc1 detector will enable to reach the physics goals at the CEPC. It should be noted that the changes to the $\mathrm{SiD}$ concept listed above are just a few possible options to reduce the cost of a detector for the CEPC energy, without compromising the physics goals at the CEPC. It is very likely that a more substantial optimization can be made after dedicated performance studies.

\section{Acknowledgments}

This research was done using resources provided by the Open Science Grid, which is supported by the National Science Foundation and the U.S. Department of Energy's Office of Science. 
The submitted manuscript has been created by UChicago Argonne, LLC, Operator of Argonne National Laboratory (Argonne). Argonne, a U.S. Department of Energy Office of Science laboratory, is operated under Contract No. DE-AC0206CH11357. Argonne National Laboratory's work was supported by the U.S. Department of Energy under contract DE-AC02-06CH11357. The U.S. Government retains for itself, and others acting on its behalf, a paid-up nonexclusive, irrevocable worldwide license in said article to reproduce, prepare derivative works, distribute copies to the public, and perform publicly and display publicly, by or on behalf of the Government.

\section{References}

1. H. Aihara et al., SiD Letter of Intent. (2009). arXiv:0911.0006 [physics.ins-det]. Presented to ILC IDAG.

2. C. Adolphsen et al., The International Linear Collider Technical Design Report - Volume 3. II: Accelerator Baseline Design (2013). arXiv:1306.6328 [physics.acc-ph] .

3. H. Abramowicz et al., The International Linear Collider Technical Design Report Volume 4: Detectors (2013). arXiv:1306.6329 [physics.ins-det].

4. M. J. Charles, PFA Performance for $\mathrm{SiD}$, in Linear colliders. Proceedings, International Linear Collider Workshop, LCWS08, and International Linear Collider Meeting, ILC08, Chicago, USA, Novermber 16-20, 2008 , (2009). arXiv:0901.4670 [physics.data-an].

5. J. S. Marshall and M. A. Thomson, Pandora Particle Flow Algorithm, in Proceedings, International Conference on Calorimetry for the High Energy Frontier (CHEF 2013), (2013), pp. 305-315. arXiv:1308.4537 [physics.ins-det].

6. N. Graf and J. McCormick, AIP Conf. Proc. 867, 503 (2006).

7. OPAL Collaboration (K. Ahmet et al.), Nucl. Instrum. Meth. A305, 275 (1991).

8. S. Chekanov, Advances in High Energy Physics 2015, 136093 (2015), Available as http://atlaswww.hep.anl.gov/hepsim/.

9. T. Sjöstrand, S. Mrenna and P. Z. Skands, J. High Energy Phys. 0605, 26 (2006).

10. R. Pordes et al., J. Phys. Conf. Ser. 78, 012057 (2007).

11. JADE Collaboration Collaboration (W. Bartel et al.), Phys. Lett B 123, 460 (1993).

12. Y. L. Dokshitzer, J. Phys. G 17, 1537 (1991), Contribution in report of the Hard QCD Working Group, Proc. Workshop on Jet Studies at LEP and HERA, Durham, December 1990. 